\title{
Direct eyelid defect closure: a prospective study of functional and aesthetic outcomes
}

\author{
Vladimir T. Thaller ${ }^{1} \cdot$ Simon N. Madge ${ }^{1,2,3} \cdot$ W. Chan $^{3} \cdot$ I. Vujic ${ }^{1} \cdot$ F. Jazayeri ${ }^{1}$
}

Received: 19 June 2018 / Revised: 7 January 2019 / Accepted: 21 February 2019 / Published online: 3 April 2019

(c) The Royal College of Ophthalmologists 2019

\begin{abstract}
Background/Objectives Direct closure (DC) of eyelid defects has been retrospectively shown to give excellent outcomes. We present prospective outcome data as further evidence to promote its wider use.

Subjects and Methods A consecutive, unselected, series of patients undergoing eyelid tumour resection was studied prospectively. DC was attempted at the time of biopsy in all of them. If DC proved impossible, delayed reconstruction using other techniques was later performed. Defect size, pre- and post-operative palpebral aperture (PA) measurements and the final visit patients' and surgeons' satisfaction scores for function and appearance were recorded.

Results Seventy-three eyelids of 70 patients were studied. Mean resected specimen width was $16.4 \mathrm{~mm}(4-26 \mathrm{~mm})$ in the DC group, versus $23.9 \mathrm{~mm}(11-42 \mathrm{~mm}$ ) for other, non-DC reconstructions. Primary DC was achieved in $74 \%$ of this cohort. Mean final post-operative PA measurements in the DC group were $0.7 \mathrm{~mm}$ vertically $(p=0.003)$ and $0.8 \mathrm{~mm}$ horizontally $(p=0.009)$ less than preoperatively, but there was no statistical difference $(p=0.1)$ in the final horizontal measurements between the operated and un-operated sides in the DC group. DC satisfaction scores were excellent for both eyelid function and appearance.

Conclusions DC of eyelid defects, irrespective of per-operative PA distortion, gives excellent late post-operative outcomes. We recommend that DC, aligning the closure tension parallel to the lid margin, is attempted whenever wound margin approximation is possible in preference to alternative reconstruction techniques, regardless of any temporary PA distortion and globe displacement that this may cause. Eyelid function and appearance recover to near normal within 2 months.
\end{abstract}

\section{Introduction}

Direct closure (DC) of eyelid defects gives excellent cosmetic and functional results following excision of lesions such as basal cell carcinoma [1, 2]. Standard oculo-plastic textbooks recommend the technique be restricted to small defects of up to a quarter of the lid length (increasing to one-third in the elderly, with or without an additional lateral

Vladimir T. Thaller

v.thaller@nhs.net

1 Royal Eye Infirmary, Derriford Hospital, Derriford Rd., Plymouth PL6 8DH, UK

2 Victoria Eye Unit, Hereford County Hospital, Hereford HR1 2ER, UK

3 South Australian Institute of Ophthalmology, Royal Adelaide Hospital, Adelaide, SA, Australia cantholysis) [2]. Presumably this restriction is because of concern about distortion of the palpebral aperture (PA) and compromise of eyelid function with DC. However, there is little in the literature documenting the outcomes of DC in larger defects, nor to justify the 'one-quarter' limit. In larger defects other techniques, including simple 'laissez-faire' spontaneous healing, a variety of flaps and the use of free grafts are recommended to restore eyelid appearance and function [3-5]. These techniques have their own complications [6].

Experience in our unit suggests that DC of much larger defects still leads to excellent final results, although to achieve DC extreme tension is often induced in the repaired eyelid, which causes temporary, sometimes profound, distortion of the PA. A previous retrospective study showed that in DC, with the tension vector appropriately directed parallel to the lid margin, there is spontaneous eyelid expansion leading to restoration of PA dimensions, lid appearance and function [1]. Here we present the first prospective DC study. 


\section{Materials and methods}

The excision of eyelid lesions under the care of the senior author (VTT) involves routine pre-operative measurement of lesion size and excision margins. It must be stressed that these measurements are linear horizontal and vertical measurements of the tissue to be resected and do not represent the curved length of lid margin to be involved in the resection. Many defects extend beyond the canthi and some do not involve the lid margin at all. All measurements are made with the tissues on gentle stretch for standardisation and are made prior to excision. Post-resection wounds naturally appear larger as their margins retract but these were not measured in this study. Pre-operative and serial post-operative horizontal and vertical PA measurements are recorded, as well as the completion of a visual analogue scale for cosmetic and functional satisfaction (independently by both patient and surgeon) at the time of the final post-operative visit (2-6 months postoperatively). Patients are routinely reviewed for removal of their post-operative eyelid dressing at 1-5 days, for possible suture removal at 1-2 weeks following surgery, and at 2-6 months for final outcome assessment.

A consecutive, unselected series of all patients undergoing excision of eyelid lesions between March 2002 and March 2007 under the Plymouth Oculo-Plastic Service forms the basis of this prospective evaluation of routine clinical practice. No patients were excluded from analysis. Standardised forms were completed for each patient, which included measurements (as above) of lesion size, specimen size and resection margins, pre-operative and post-operative vertical and horizontal PAs, as well as surgical details and copies of post-operative visual analogue satisfaction scales. In addition, a surrogate marker of symmetry between the operated and un-operated sides was calculated by subtracting the vertical or horizontal PA measurement of the operated side from that of the un-operated side.

DC of eyelid defects was initially attempted in all patients, irrespective of the defect size or of the lid tension or globe displacement that it caused. Where DC of full thickness eyelid margin defects was possible, the posterior lamella was reconstructed by end-to-end suturing of the remaining tarsal plate with $6 / 0$ braided, uncoated, polyglycolic acid sutures. In the absence of tarsal plate at one end, the cut edge of the remaining tarsal plate was sutured to the canthal tendon stump or to orbital rim periosteum, with the same type of suture. The lid margin and anterior lamella were closed either with the same suture material or, more commonly, 6/0 silk. Where the surgical defect did not involve the eyelid margin, the wound tension was taken up by buried 4/0 braided, uncoated, polyglycolic acid sutures placed in the subcutaneous tissues. These facilitated easier skin closure with less skin tension. The wounds were
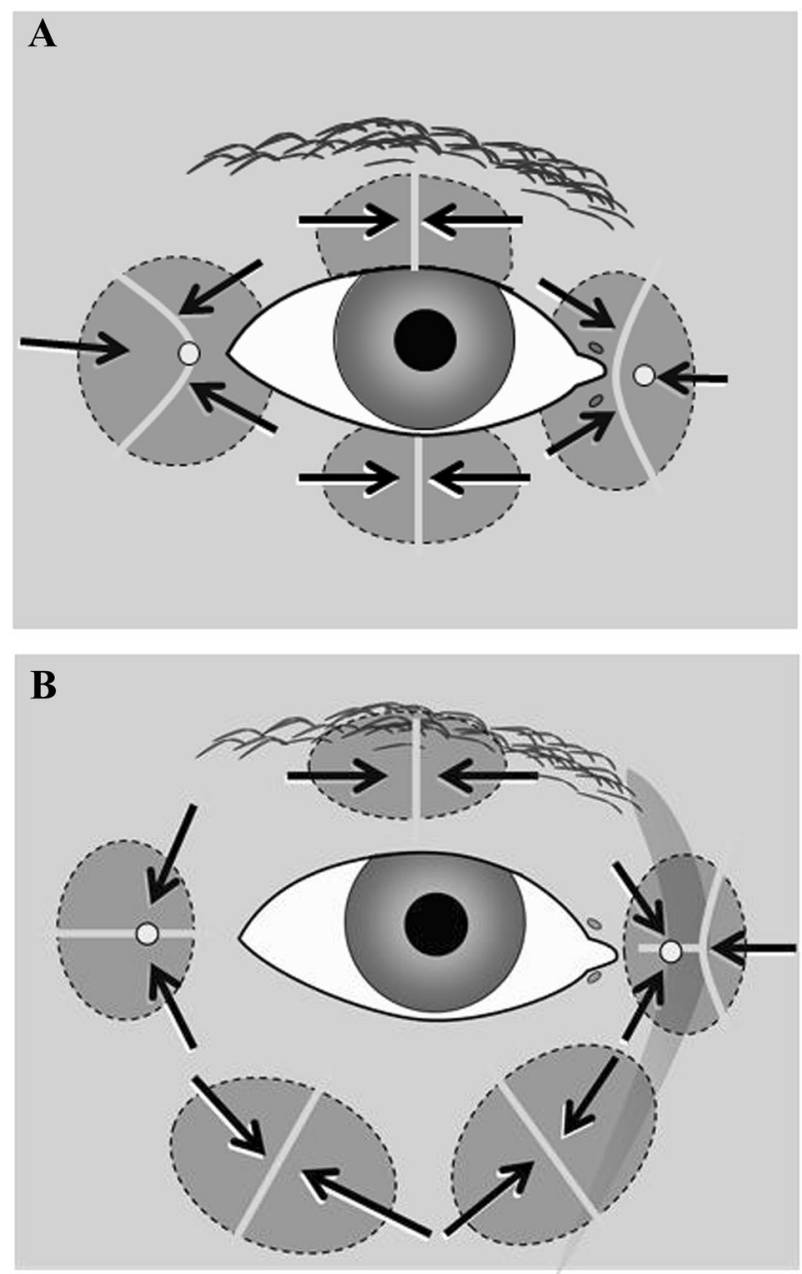

Fig. 1 Desired closure tension vectors (black arrows) and resulting scar orientation (grey lines) for lid margin defects (a) and for defects not involving the lid margin (b)

always closed to direct the tension vector between the canthal tendon to orbital rim insertion points (the posterior lacrimal crest medially and Whitnall's tubercle laterally). This meant that the closure induced tension was parallel to the lid margin and the resultant scar perpendicular to the margin. This is critical to avoid impairing eyelid function or causing ectropion (Fig. 1).

Where lid defects were closed under extreme tension, full thickness tarsal plate sutures were often employed, as opposed to the more typical partial thickness sutures normally used [3]. Under such tension, post-operative dehiscence of the wound occasionally occurred, in which circumstance the repair effectively became a 'directed' or partial 'laissez-faire', often still with excellent results. For patients in whom DC was initially possible, but who subsequently had wound dehiscence, their results were analysed on an intention-to-treat basis, with PA measurements and satisfaction scores reflecting the outcome of the initially selected technique. No 'undermining' of tissue was 
performed in any case that underwent DC, be the defect on the lid margin or elsewhere. Cantholysis was not performed in any case, the rationale being that inducing tension in the eyelid is crucial in stimulating expansion of the eyelid tissues and a return towards normal dimensions [1]. No additional tissue was excised to prevent 'dog ears'.

In patients where DC was not possible, reconstruction involved 'laissez-faire' spontaneous healing (often assisted with strategically placed sutures to minimise defects, i.e., 'directed laissez-faire'), local skin flaps, free skin grafts and occasionally the use of cross-linked porcine dermal collagen (Permacol, Covidien, Ireland) in combination with skin flaps.

As this was a non-interventional observational study of routine clinical practice, ethical approval was not required.

\section{Results}

Seventy-three eyelids of 70 patients were included in the study Table 1 . The mean age of the patients at the time of surgery was 70 years (range 31-91) with an almost equal gender distribution (36 males, 34 females). The confirmed histological diagnosis was basal cell carcinoma in 62 cases, squamous cell carcinoma in 2 , sebaceous cell carcinoma in 1 , naevus in 3 and other non-malignant lesions in 5 cases.

The lesion per se involved the lower lid only in 50 cases, the upper lid in 13, the canthus and either lid in 6 and purely the canthus in 2 (exact documentation absent in 2 cases). The resection margin involved either canthus in 31 cases, being confined to the lid only in 40 . The lid margin was involved in the resection in 60 cases. Excision margins around the lesion were $4 \mathrm{~mm}$ in 60 cases and $2 \mathrm{~mm}$ in 11 (inadequate documentation 2 cases).

The mean horizontal width of the resected specimen was $18.2 \mathrm{~mm}$ (range 4-42 mm), with a mean height of $12.8 \mathrm{~mm}$ (range $4-55 \mathrm{~mm}$ ). The sizes of the specimens among patients undergoing and not undergoing DC are shown in Table 2. The mean ratio of specimen width to pre-operative horizontal PA measurement was 0.61 (range 0.14-1.04) in the group who underwent DC compared with 0.95 (range $0.39-1.56)$ for other surgical techniques.

Following excision biopsy, DC was possible in 54 $(74 \%)$. Directed 'laissez-faire' was used in seven cases $(10 \%)$, the use of a graft or flap in eight $(11 \%)$, and the combination of cross-linked porcine dermal collagen with a local skin flap in four (5\%). Reconstruction was delayed in 21 cases (pending confirmation of histological clearance), with immediate attempts to close the defect (or deciding to pursue 'laissez-faire') being made in 52 cases. Of the 54 patients who underwent DC, four developed a degree of post-operative wound dehiscence, which ranged in size from 2 to $6 \mathrm{~mm}$. One of these patients developed a small lateral notch in the lid margin and another had a small skin web between lateral canthus and remaining tarsal plate, akin to the appearance following surgical cantholysis; the remaining two patients healed well.

There were no significant differences between the operated and un-operated eyelids in horizontal or vertical PA measurements prior to surgery (paired $t$-test, $p=0.68$ and $p=0.82$, respectively).

In eyes that underwent $\mathrm{DC}$, the final post-operative vertical PA measurements (mean $8.6 \mathrm{~mm} \pm 0.2 \mathrm{~mm}$ ) and horizontal PA measurements (mean $26.1 \mathrm{~mm} \pm 0.3 \mathrm{~mm}$ ) were statistically less than the pre-operative measurements (mean $9.3 \mathrm{~mm} \pm 0.21 \mathrm{~mm}$ and $26.9 \mathrm{~mm} \pm 0.3 \mathrm{~mm}$, respectively) ( $t$-test, $p=0.003$ and $p=0.009$ respectively) (Table 2). In addition, horizontal and vertical PA measurements at both first dressing and 1-2 weeks following surgery were significantly different from pre-operative measurements $(p=0.00, p=0.00$ and $p=0.03$, and $p=0.00$ respectively).

In the DC group, there was no statistical difference between the final horizontal PA measurements for the operated and un-operated eyes $(25.8 \mathrm{~mm}$ versus $26.1 \mathrm{~mm}$, $p=0.10, t$-test), but there was a difference in the vertical PA measurements $(8.5 \mathrm{~mm}$ versus $8.8 \mathrm{~mm}, p=0.02)$ (Table 2).

The satisfaction scores in each category (both eyelid function and appearance, as rated independently by patient and surgeon) were not normally distributed, but were negatively skewed. Statistical comparison between the two groups would not be appropriate as they were not randomised and involved different initial defect sizes. The nonDC closure data are merely presented to provide context to the satisfaction scores.

Figures 2-5 illustrate some typical results obtained after DC.

\section{Discussion}

Direct eyelid defect closure (DC) is well established as the preferred eyelid reconstruction technique, its advantages being self-evident $[1,2]$. However, its application is traditionally restricted to eyelid margin defects measuring less than a quarter of the horizontal PA (or one-third when combined with a lateral cantholysis) $[4,7,8]$ for fear of distorting the PA and compromising lid function, even though little published evidence supports such restriction and fears. This prospective study broadly corroborates the results of a previous retrospective study of DC of lid defects (ranging from 29 to $108 \%$ of the horizontal PA), which showed that the final postoperative PA measurements were not significantly different between the operated and un-operated sides [1]. That retrospective study differed from our current one in that it 


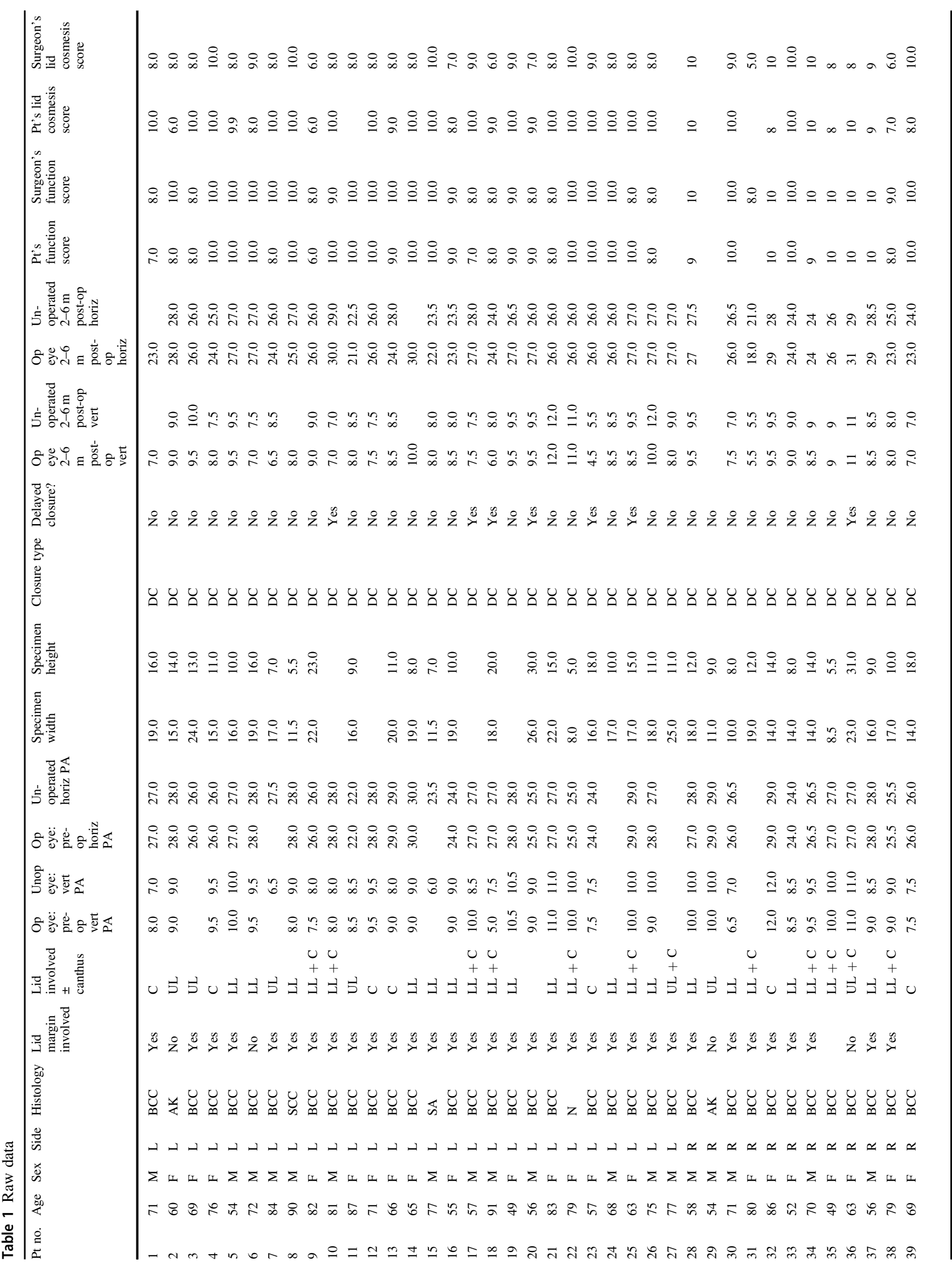




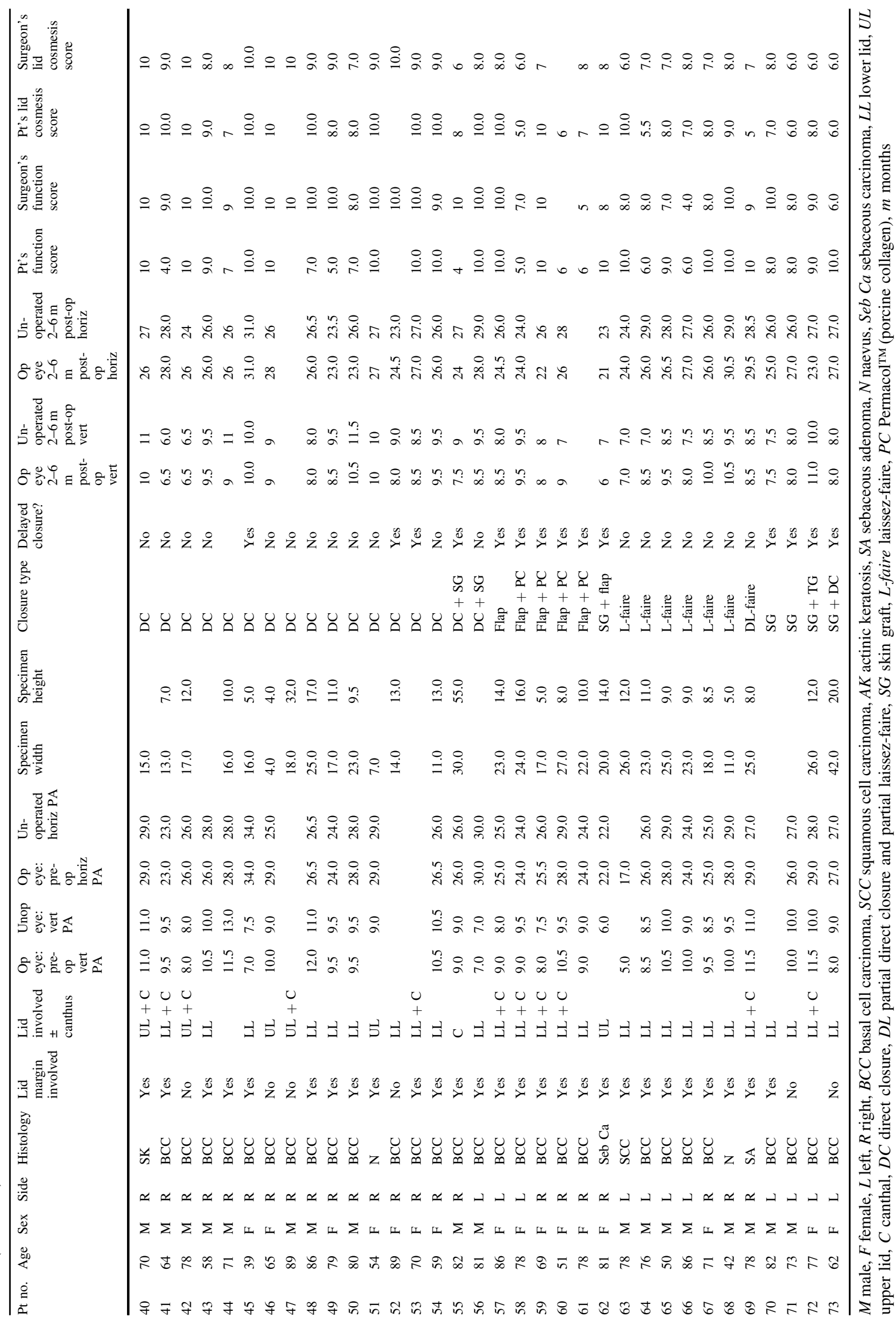


Table 2 Data summary

Lid defect sizes $(\mathrm{mm})$ for patients undergoing direct closure (DC) and non-direct closure (non-DC) techniques

\begin{tabular}{ll}
\hline DC group & $\begin{array}{l}\text { Non-DC } \\
\text { group }\end{array}$ \\
\hline
\end{tabular}

Initial defect size

(mm)

Height mean (range) $12.6(4-32)$

$13.5(5-55)$

Width mean (range) 16.4 (4-26)

$23.9(11-42)$

Pre- and post-DC palpebral aperture measurements

$\begin{array}{llll}\begin{array}{l}\text { Pre- } \\ \text { operative }\end{array} & \begin{array}{l}\text { Post- } \\ \text { operative }\end{array} & \Delta & p\end{array}$

Palpebral aperture

( $\mathrm{mm})$

$\begin{array}{lllll}\text { Vertical PA } & 9.3 & 8.6 & -0.7 & 0.003 \\ \text { Horizontal PA } & 26.9 & 26.1 & -0.8 & 0.009\end{array}$

Operated vs un-operated palpebral aperture symmetry

$\begin{array}{lllll} & \text { DC final } & \begin{array}{l}\text { Contra } \\ \text { lateral }\end{array} & \boldsymbol{\Delta} & \boldsymbol{p} \\ \text { Vertical PA (mm) } & 8.5 & 8.8 & -0.3 & 0.02 \\ \text { Horizontal PA (mm) } & 25.8 & 26.1 & -0.3 & 0.1\end{array}$

Mean satisfaction scores for patients' and surgeon's assessment of eyelid function and cosmesis $(1=$ poor, $10=$ excellent $)$

\section{Direct closure group}

\begin{tabular}{lll} 
Patient—eyelid & 9 & 8.3 \\
function & & 7.7 \\
Patient—cosmesis & 9.3 & 8.2 \\
$\begin{array}{l}\text { Surgeon—eyelid } \\
\text { function }\end{array}$ & 9.4 & \\
Surgeon-cosmesis & 8.6 & 7.1 \\
\hline
\end{tabular}

was based on late post-operative photographs assessed by a blinded observer looking for asymmetry between the two eyes. The present prospective study compared both pre- and post-operative clinical PA measurements on the treated side, as well as the final disparity between the operated and unoperated PAs. In contrast to the previous study, we did find that the PA measurements following DC are statistically smaller than preoperatively (both vertically and horizontally) but that the absolute final differences are small (0.7 and 0.8 $\mathrm{mm}$, respectively), too small to have been reliably picked up by the photographic analysis in the quoted previous study. These differences are arguably clinically insignificant as evidenced by the patients' own cosmesis satisfaction scores in the current study (Table 2). The absence of a statistically significant difference between the final post-operative horizontal PA measurements of the operated compared with the un-operated sides in the patients undergoing DC supports this view. This discrepancy between PA change and inter eye asymmetry might be explained by a degree of pre-operative tissue expansion of the affected lid caused by the mass effect of the lid tumour (as in 'mechanical ectropion'), e.g., Fig. 3.

It should be emphasised that, as stated in the Materials and methods section, the measurements refer to the maximal horizontal and vertical dimensions of the tissue removed and not to the actual lid margin involved. The percentages quoted are relative to the horizontal PA, which is a straight line measurement, and are not a percentage of the curved lid margin length, the latter obviously being the longer. Thus, this methodology gives higher percentage values than might seem intuitive.

DC was achievable in $74 \%$ in this prospective, unselected series of patients, suggesting that it could potentially
Fig. 2 a (Patient 3) Upper lid $\mathrm{BCC}$ with $4 \mathrm{~mm}$ margin markings $(24 \times 13 \mathrm{~mm}$ specimen $\mathrm{W} \times \mathrm{H})$. b On table, direct defect closure under tension (patient unable to open eye). $\mathbf{c}, \mathbf{d}$ At 4 months, open and closed respectively, i.e., full eyelid function
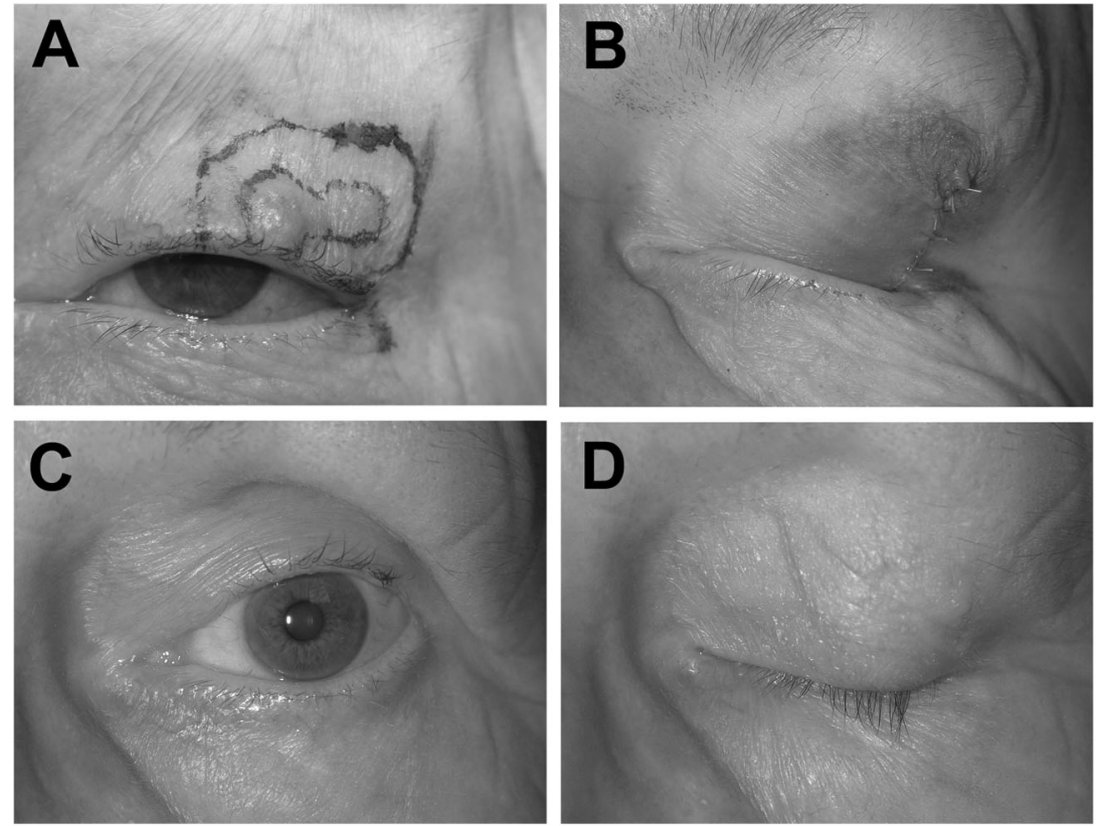

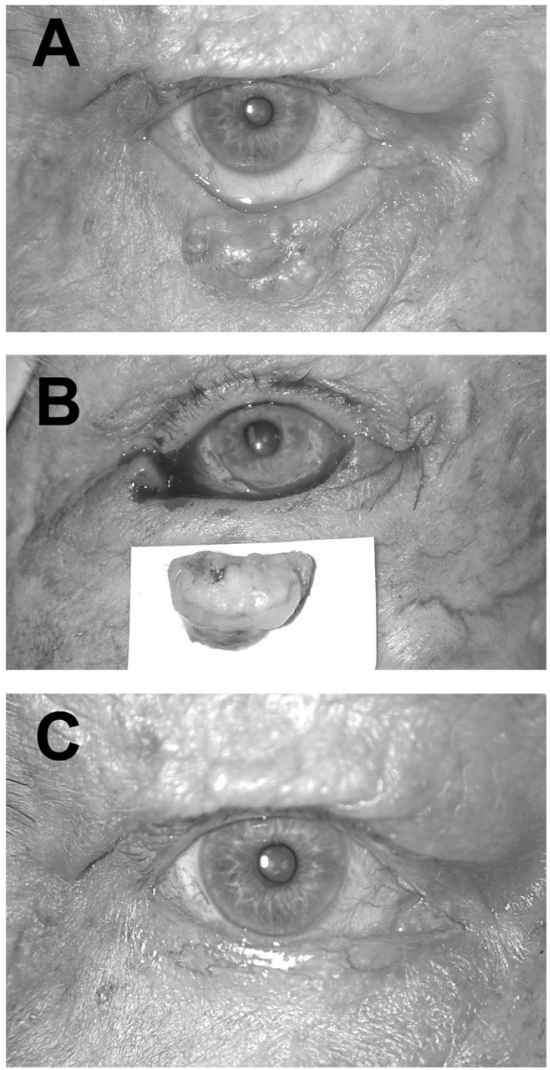

Fig. 3 a (Patient 48) Lower lid BCC with secondary mechanical ectropion. b Specimen $(25 \mathrm{~mm} \times 17 \mathrm{~mm}, \mathrm{~W} \times \mathrm{H})$ and lid margin defect. c Final appearance at 4 months after DC, showing normal lid margin

be used in more patients than textbooks recommend. This proportion is similar to that reported elsewhere $[9,10]$. There was a large overlap between the DC and non-DC group defect sizes indicating that defect size per se should not be used to decide, which patients are suitable for DC (Table 2). If the wound edges can be pulled together in the appropriate direction (Fig. 1) at the time of surgery then DC is an option, irrespective of measured defect size or the tension required to bring the edges together.

Important benefits of the DC technique include a normal lid margin appearance with uniform thickness (Figs. 2-5). There is no lateral margin thinning as is seen following cantholysis, an absence of a red lid margin, often seen after posterior lamellar reconstruction [11], as well as the lack of skin hair contact with the cornea, commonly noted after lid margin skin grafting. There is a continued presence of eyelashes and meibomian glands, albeit slightly spaced out. Because we did not perform cantholysis in any patient lateral canthal webbing was observed in only one instance. Finally as there is no tissue donor site, no additional scars and associated morbidity are created. The ultimate postoperative results after DC in this series are excellent as the satisfaction scores (both patients' and surgeon's) indicate with near-normalisation of PA measurements.
The present study does not compare the outcomes of DC with other techniques as the two groups in this study were mutually exclusive and therefore not comparable. Some might postulate that other techniques that recruit extra tissue, such as flaps and grafts, lead to less alteration in the PA measurements than the small reduction seen with the DC technique. This possibility has not been addressed by this non-randomised study.

The apparent absurdity of some specimens measuring $>100 \%$ of the PA dimensions is explained by some tumours extending beyond the canthi and by the geometry of a curved lid margin compared with the straight horizontal PA reference measurement.

The sizes of many of the defects closed by DC in this study are larger than conventional teaching allows $[2,3]$. Closure of such defects often required extreme tension, sometimes with a consequent temporary entropion. In the immediate aftermath of surgery, the PAs were often markedly smaller and distorted. DC of larger lid defects often causes a temporary complete ptosis as the induced lid margin tension displaces the globe upwards under the upper lid. In no case did this persist. We postulate that the displaced eye functions as a tissue expander [1], providing the steady, sustained force necessary for eyelid expansion [1214]. In most cases, PA dimensions returned to nearly their final measurements by only 1-2 weeks following surgery, the rapid tissue expansion allowing eyelid opening in most cases (where measured) at this stage.

That significant eyelid tissue and specifically eyelid margin expansion can occur should come as no surprise to eye surgeons who observe it in every patient who develops ectropion or the floppy lid syndrome: the lid margin becoming too long for purpose and requiring shortening as part of the surgical correction. This expansion is particularly marked in cicatricial ectropion due to chronic sustained anterior lamellar traction. The DC of eyelid defects under appropriately directed tension merely makes use of this phenomenon. The vector of this closure tension is critical. It must be directed between the medial and lateral eyelid to orbital rim fixation points (insertions of the canthal tendons) (Fig. 1). This vector is effectively at 90 degrees to Langer's lines. The tension is lost if cantholysis is performed so, contrary to common teaching, this should never be carried out as an adjunct to DC or the desired expansion will simply not occur and lid margin thinning and lateral canthal rounding will ensue.

Dehiscence of eyelid wounds was seen in 4 of the 54 DC patients (7\%), with consequent effects on the final lid margin in two. However, we would argue that such results are certainly as good as, if not better than 'intended' laissezfaire from the outset; certainly, the satisfaction scores of these two patients compared favourably with the laissezfaire group. As this was a study of routine clinical practice, 
Fig. 4 a (Patient 7) Lesion and $4 \mathrm{~mm}$ margins (Specimen $17 \times$ $7 \mathrm{~mm}, \mathrm{~W} \times \mathrm{H}$ ). b Upper lid excision defect. c Deep suture placement.d End of surgery DC.

Patient unable to open eye.

e Open and $\mathbf{f}$ closed operated eye at 2 months

Fig. 5 a (Patient 10) Two adjacent $\mathrm{BCCs}$ giving rise to (b) a single large defect. c Oneweek follow-up DC appearance. d DC appearance at 3 years
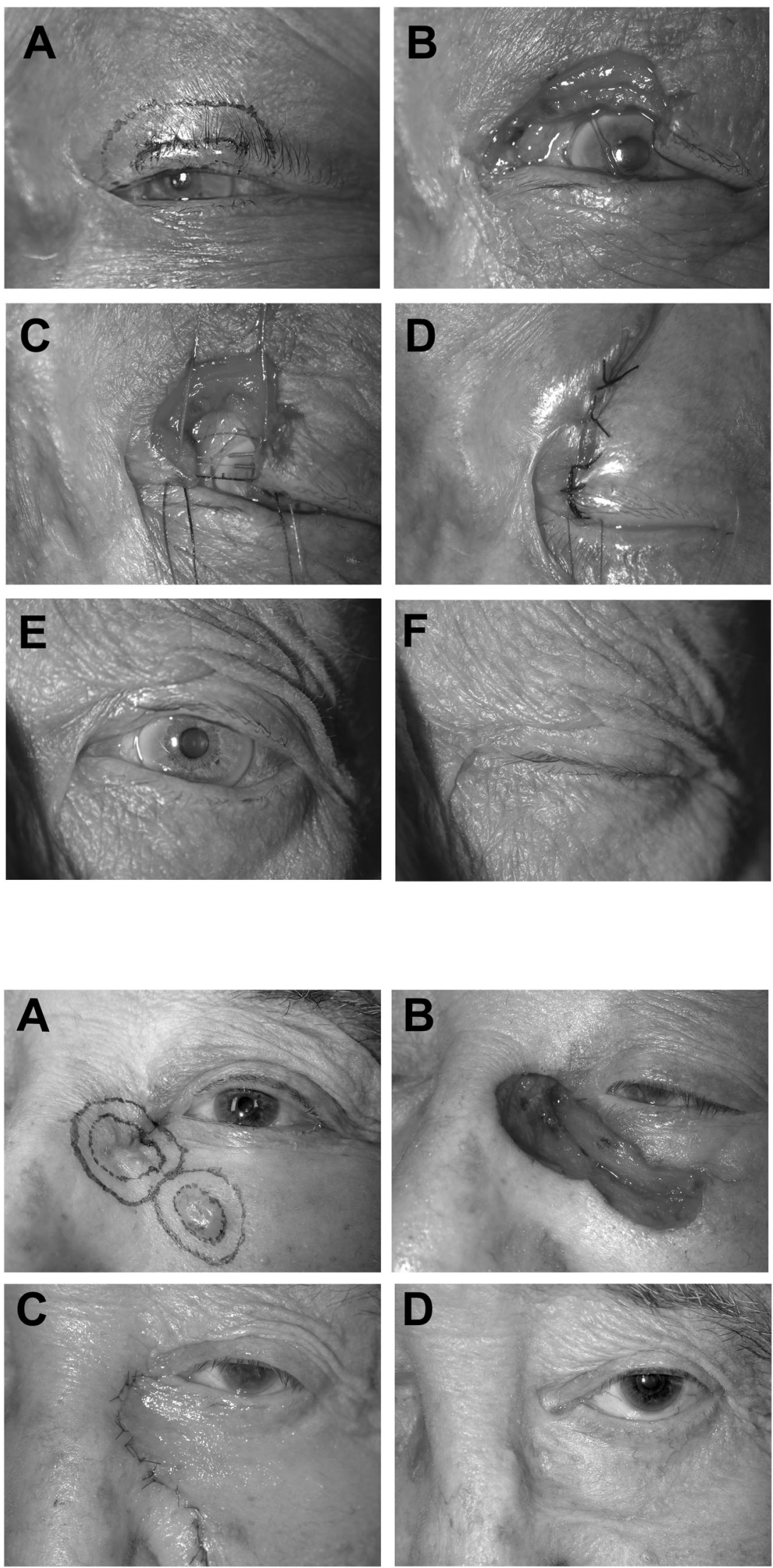
results were deliberately analysed on an intention-to-treat basis and the dehiscences included within the DC group for scientific rigour.

Patients should be counselled that the early induced post-operative distortion of the PA is temporary and should not cause them alarm and that the final cosmetic and functional results will be excellent. The learning curve for DC is short as direct wound closure is part of every surgeon's skill set.

One step DC at the time of tumour excision need not be delayed until histology results are available to confirm tumour clearance. Should subsequent tumour re-excision be necessary the anatomy remains clear as no undermining has been undertaken and no grafts or flaps need to be sacrificed. The resulting DC linear scar includes all the previous excision margins so can easily be re-excised with additional margins if required as the histology dictates.

The strength of this study is that it was a prospective evaluation of DC outcomes in an unselected series of patients. Such outcomes have not been reported previously. We acknowledge that a masked, comparative, randomised, comparison between DC and standard practice would be more robust and that ours was a single surgeon series. A randomised study needs to be carried out by someone sceptical of DC. We ourselves, with our long positive experience of the benefits of DC, could not ethically justify randomisation.

In conclusion, the results presented support our contention that DC, with appropriately directed eyelid tension, should be attempted as the repair of choice for all lid defects. Should DC be unachievable on the operating table or should the ultimate result of DC be judged unsatisfactory nothing will have been lost; all the alternative reconstruction options remain. This should reassure any surgeons wishing to expand their use of DC. No delayed secondary reconstruction was required in this series (not even for dehiscence). The patients' subjective assessments of final eyelid appearance and function following DC are good. Surely such satisfaction is the ultimately goal of any reconstruction?

\section{Summary}

\section{What was known before}

- Direct closure (DC) is effective.

- DC, even of large eyelid defects, results in excellent eyelid function, appearance and symmetry.

- Eyelids expand under tension.

- DC created tension promotes eyelid tissue expansion.
What this study adds

- Prospective DC evaluation in an unselected series of lid tumour patients.

- DC was possible in three-quarters of them.

- DC gives high satisfaction with the function and cosmesis of the reconstructed lid.

- Diagrammatic guidance on DC tension vector orientation (at 90 degrees to Langer's lines).

\section{Compliance with ethical standards}

Conflict of interest The authors declare that they have no conflict of interest.

Publisher's note: Springer Nature remains neutral with regard to jurisdictional claims in published maps and institutional affiliations.

\section{References}

1. Thaller VT, Then KY, Luhishi E. Spontaneous eyelid expansion after full thickness eyelid resection and direct closure. Br J Ophthalmol. 2001;85:1450-4.

2. Kakizaki H, Madge SN, Mannor G, Selva D, Malhotra R. Oculoplastic surgery for lower eyelid reconstruction after periocular cutaneous carcinoma. Int Ophthal Clinics. 2009;49:143-55.

3. Collin JRO. A manual of systematic eyelid surgery. 3rd ed. London, UK: Elsevier; 2006. p. 115-45.

4. Moss AL, Cooper MA, Lendrum J, Hiles RW. The sanctity of the upper lid in lower eyelid reconstruction questioned. A modification of a lid sharing procedure with a long-term follow-up. Br J Plast Surg. 1987;40:246-57.

5. Putterman AM. Reconstruction of the eyelids following resection for carcinoma. Clin Plast Surg. 1985;12:393-410.

6. Hawes MJ, Jamell GA. Complications of tarsoconjunctival grafts. Ophthal Plast Reconstr Surg. 1996;12:45-50.

7. Rathbun JE. Eyelid surgery. 1 ed. Boston, USA: Little Brown; 1990.

8. Stasior GO, Stasior OG. Prevention and management of complications. In: Dortzbach RK, editor. Ophthalmic plastic surgery. 1 ed. New York, USA: Raven Press; 1994. p. 125-40.

9. Chadha V, Wright M. Small margin excision of periocular basal cell carcinomas. Br J Ophthalmol. 2009;93:803-6.

10. Hamada S, Kersey T, Thaller VT. Eyelid basal cell carcinoma: non-Mohs excision, repair, and outcome. Br J Ophthalmol. 2005;89:992-4.

11. Hawes MJ, Jamell GA. Complications of tarsoconjunctival grafts. Ophthal Plast Reconstr Surg. 1996;12:45-50.

12. Neumann CT. The expansion of an area of skin by progressive distension of a subcutaneous balloon. Plastic Reconstr Surg. 1957;19:124-30.

13. Johnson TM, Lowe L, Brown MD, Sullivan MJ, Nelson BR. Histology and physiology of tissue expansion. J Dermatol Surg Oncol. 1993;19:1074-8.

14. Foster JA, Scheiner AJ, Wulc AE, Wallace IB, Greenbaum SS. Intraoperative tissue expansion in eyelid reconstruction. Ophthalmology. 1998;105:170-5. 